\section{Composite Adaptation for Neural Network-Based Controllers}

Parag M. Patre, Shubhendu Bhasin, Zachary D. Wilcox, and Warren E. Dixon

\begin{abstract}
With the motivation of using more information to update the parameter estimates to achieve improved tracking performance, composite adaptation that uses both the system tracking errors and a prediction error containing parametric information to drive the update laws, has become widespread in adaptive control literature. However, despite its obvious benefits, composite adaptation has not been widely implemented in neural network-based control, primarily due to the neural network (NN) reconstruction error that destroys a typical prediction error formulation required for the composite adaptation. This technical note presents a novel approach to design a composite adaptation law for NNs by devising an innovative swapping procedure that uses the recently developed robust integral of the sign of the error (RISE) feedback method. Semi-global asymptotic tracking is proven for a Euler-Lagrange system. Experimental results are provided to illustrate the concept.
\end{abstract}

\section{INTRODUCTION}

Euler-Lagrange (EL) dynamics can be used to represent a number of practical and contemporary engineering systems. As such, nonlinear EL systems serve as a benchmark for nonlinear control research [1]. Within this domain of research, the effects of uncertainty and disturbances in the dynamics continue to be a focal point. In particular, neural networks (NNs) have found a widespread use over the last decade as a nonmodel-based feedforward control element (cf. some pioneering works in [2]-[11]) to approximate and compensate for uncertainties that are not linear-in-the-parameters (i.e., non-LP). The ability of NNs to compensate for non-LP uncertainty is due to the Universal Approximation Property [2]-[4] that states any sufficiently smooth function can be approximated by a suitable large network for all inputs in a compact set, and the resulting function reconstruction error is bounded. The NN weight estimates are generated using adaptation laws that are designed to cancel the cross terms in the Lyapunov stability analysis which leads to an adaptation law structure that uses the system tracking errors to update the weights. Ideally, the adaptation laws would include some estimate of the actual mismatch between the unknown function and its $\mathrm{NN}$ approximation to improve the $\mathrm{NN}$ estimation. To inject some measure of the adaptation error in the update law, standard adaptive control utilizes a swapping procedure [12]-[17] to design a measurable prediction error that directly relates to the parameter mismatch. The prediction error is defined as the difference between the predicted parameter estimate value and the actual system uncertainty. The advantages of improved tracking control potentially enabled by prediction error based adaptive update laws led to several results that use either the prediction error or a composite [16] of the prediction error and the tracking error (cf. [16]-[19] and the references within).

Manuscript received March 29, 2009; revised September 11, 2009 and September 14, 2009. First published February 02, 2010; current version published April 02, 2010. This work was supported in part by the NSF CAREER Award 0547448, NSF Award 0901491, and the Department of Energy under Grant DE-FG04-86NE37967 as part of the DOE University Research Program in Robotics (URPR). Recommended by Associate Editor A. Astolfi.

The authors are with the Department of Mechanical and Aerospace Engineering, University of Florida, Gainesville, FL 32611 USA (e-mail: parag. patre@gmail.com; sbhasin@ufl.edu; zibrus@gmail.com; wdixon@ufl.edu).

Color versions of one or more of the figures in this technical note are available online at http://ieeexplore.ieee.org.

Digital Object Identifier 10.1109/TAC.2010.2041682
However, the swapping procedure [20] used in standard adaptive control cannot be extended to NN controllers directly. The presence of a NN reconstruction error has impeded the development of composite adaptation laws for NNs. Specifically, the reconstruction error gets filtered and included in the prediction error destroying the typical prediction error formulation. Using discontinuous sliding mode feedback, the first composite adaptation method developed for a NN-based controller is given in [21], [22]. The approach in [21], [22] focuses on a single-layer $\mathrm{NN}$, where the adaptive control problem is formulated in a manner similar to [23]. Then the dead zone adaptation method from [23] is applied to compensate the disturbance terms in the prediction error. The use of dead zone adaptation implies that the update law is composite only for part of the control operation, when the prediction error norm lies outside the dead zone, which is determined by the size of the NN residual error. Thus, if the NN residual error is larger than the prediction error, the method in [21] and [22] cannot use composite adaptation. Using a similar dead zone adaptation-based approach, a composite adaptation method was also developed for locally weighted learning in [24] using a continuous feedback. However, the approach in [24] requires measurement of the state derivative and yeilds a uniformly ultimately bounded tracking result.

With the motivation of achieving improved performance (inspired by the seminal work in [16] for traditional adaptive control methods), this technical note presents a novel approach to develop a prediction error-based composite adaptive NN controller for an EL system using the recent continuous robust integral of the sign of the error (RISE) [25] technique that was originally developed in [26] and [27]. The RISE architecture is adopted since this method can accommodate for $C^{2}$ disturbances and yield asymptotic stability. The RISE technique was used in [28] to prove the first ever asymptotic result for a NN-based controller using a continuous feedback. In this technical note, the RISE feedback is used in conjunction with a NN feedfoward element similar to [28], however, unlike the typical tracking error-based gradient update law used in [28], the result in this technical note uses a composite update law driven by both the tracking and the prediction error. As opposed to dead zone adaptation [21], [22] to compensate for the effect of NN reconstruction error, an innovative use of the RISE structure is also employed in the prediction error update (i.e., the filtered control input estimate). Sufficient gain conditions are derived using a Lyapunov-based stability analysis under which this unique double-RISE control strategy yields a semi-global asymptotic stability for the system tracking errors and the prediction error, while all other signals and the control input are shown to be bounded. Since a multi-layer NN includes the first layer weight estimate inside a nonlinear activation function, proving that the NN weight estimates are bounded is a challenging task. A projection algorithm is used to guarantee the boundedness of the weight estimates. However, if instead a single-layer $\mathrm{NN}$ is used, projection is not required and the weight estimates can be shown bounded via the stability analysis. The control development in this technical note can be easily simplified for a single-layer $\mathrm{NN}$ by choosing a fixed set of suitable first layer weights. Moreover, the control development can be extended for higher order dynamic systems similar to [29], [30]. Experimental results are presented that demonstrate improved tracking performance for the proposed composite NN law as compared to a typical gradient-based NN update law.

\section{DYNAMIC SYSTEM} form:

Consider a class of second-order nonlinear systems of the following

$$
\ddot{x}=f(x, \dot{x})+G(x) u
$$


where $x(t), \dot{x}(t) \in \mathbb{R}^{n}$ are the system states, $u(t) \in \mathbb{R}^{n}$ is the control input, $f(x, \dot{x}) \in \mathbb{R}^{n}$ and $G(x) \in \mathbb{R}^{n \times n}$ are unknown nonlinear $\mathcal{C}^{2}$ functions. Throughout the technical note, $|\cdot|$ denotes the absolute value of the scalar argument, $\|\cdot\|$ denotes the standard Euclidean norm for a vector or the induced infinity norm for a matrix, and $\|\cdot\|_{F}$ denotes the Frobenius norm of a matrix. The following properties and assumptions will be exploited in the subsequent development. A. 1: $G(\cdot)$ is symmetric positive definite, and satisfies the following inequality $\forall \xi(t) \in \mathbb{R}^{n}:$

$$
\underline{g}\|\xi\|^{2} \leq \xi^{T} G^{-1} \xi \leq \bar{g}(x)\|\xi\|^{2}
$$

where $g \in \mathbb{R}$ is a known positive constant, and $\bar{g}(x) \in \mathbb{R}$ is a known bounded positive function such that $\bar{g}(x)<\bar{g}$ for some $\bar{g}>0$. A. 2: the functions $G^{-1}(\cdot)$ and $f(\cdot)$ are locally Lipschitz and second order differentiable such that $G^{-1}(\cdot), \dot{G}^{-1}(\cdot), \ddot{G}^{-1}(\cdot), f(\cdot), \dot{f}(\cdot), \ddot{f}(\cdot) \in$ $\mathcal{L}_{\infty}$ if $x^{(i)}(t) \in \mathcal{L}_{\infty}, i=0,1,2,3$, where $(\cdot)^{(i)}(t)$ denotes the $i^{t h}$ derivative with respect to time. A. 3: the desired trajectory $x_{d}(t) \in$ $\mathbb{R}^{n}$ is designed such that $x_{d}^{(i)}(t) \in \mathcal{L}_{\infty}, i=0,1, \ldots, 4$ with known bounds.

\section{CONTROL OBJECTIVE}

The objective is to design a continuous composite adaptive [16] $\mathrm{NN}$ controller which ensures that the system state $x(t)$ tracks a desired time-varying trajectory $x_{d}(t)$ despite uncertainties in the dynamic model. To quantify this objective, a tracking error, denoted by $e_{1}(t) \in \mathbb{R}^{n}$, is defined as

$$
e_{1} \triangleq x_{d}-x
$$

To facilitate the subsequent analysis, filtered tracking errors, denoted by $e_{2}(t), r(t) \in \mathbb{R}^{n}$, are also defined as

$$
e_{2} \triangleq \dot{e}_{1}+\alpha_{1} e_{1}, \quad r \triangleq \dot{e}_{2}+\alpha_{2} e_{2}
$$

where $\alpha_{1}, \alpha_{2} \in \mathbb{R}$ denote positive constants. The subsequent development is based on the assumption that the system states $x(t), \dot{x}(t)$ are measurable. Hence, the filtered tracking error $r(t)$ is not measurable since the expression in (4) depends on $\ddot{x}(t)$.

\section{CONTROL DEVElopMENT}

The open-loop tracking error system is developed by premultiplying (4) by $G^{-1}(x)$ and utilizing the expressions in (1), (3), (4) as

$$
G^{-1}(x) r=\psi+S_{1}-u .
$$

In (5) $\psi\left(x_{d}, \dot{x}_{d}, \ddot{x}_{d}\right) \in \mathbb{R}^{n}$ is defined as

$$
\psi \triangleq G_{d}^{-1} \ddot{x}_{d}-G_{d}^{-1} f_{d}
$$

where $G_{d}^{-1}\left(x_{d}\right) \triangleq G^{-1}\left(x_{d}\right)$ and $f_{d}\left(x_{d}, \dot{x}_{d}\right) \triangleq f\left(x_{d}, \dot{x}_{d}\right)$. Also in (5), the auxiliary function $S_{1}(x, \dot{x}, t) \in \mathbb{R}^{n}$ is defined as

$$
S_{1} \triangleq G^{-1} \alpha_{2} e_{2}+G^{-1} \ddot{x}_{d}-G_{d}^{-1} \ddot{x}_{d}-G^{-1} f+G_{d}^{-1} f_{d}+G^{-1} \alpha_{1} \dot{e}_{1} \text {. }
$$

The unknown dynamics in (6) can be represented by a three-layer NN as [3], [4]

$$
\psi=W^{T} \sigma\left(V^{T} \bar{x}_{d}\right)+\varepsilon\left(\bar{x}_{d}\right) .
$$

In (8), $V \in \mathbb{R}^{\left(N_{1}+1\right) \times N_{2}}$ and $W \in \mathbb{R}^{\left(N_{2}+1\right) \times n}$ are bounded constant ideal weight matrices for the first-to-second and second-to-third layers respectively, where $N_{1}$ is the number of neurons in the input layer, $N_{2}$ is the number of neurons in the hidden layer, and $n$ is the number of neurons in the third layer. The activation function in (8) is denoted by $\sigma(\cdot) \in \mathbb{R}^{N_{2}+1}, \varepsilon\left(\bar{x}_{d}\right) \in \mathbb{R}^{n}$ is the functional reconstruction error, and $\bar{x}_{d}(t) \in \mathbb{R}^{3 n+1}$ is the input vector defined as $\bar{x}_{d}(t) \triangleq\left[1 x_{d}^{T}(t) \dot{x}_{d}^{T}(t) \ddot{x}_{d}^{T}(t)\right]^{T}$ so that $N_{1}=3 n$. Note that, augmenting the input vector $\bar{x}_{d}(t)$ and activation function $\sigma(\cdot)$ by " 1 " allows us to have thresholds as the first columns of the weight matrices [3], [4]. Thus, any adaptation of $W$ and $V$ then includes adaptation of thresholds as well. The function reconstruction error and its first two time derivatives (i.e., $\varepsilon\left(\bar{x}_{d}\right), \dot{\varepsilon}\left(\bar{x}_{d}, \dot{\bar{x}}_{d}\right)$, and $\left.\ddot{\varepsilon}\left(\bar{x}_{d}, \dot{\bar{x}}_{d}, \ddot{\bar{x}}_{d}\right)\right)$ are assumed to be bounded by known constants. For a NN control development with unknown bounds of the residual error, please see the approach in [31]. Based on (8), the typical three-layer NN approximation for $\psi\left(\bar{x}_{d}\right)$ is given as [3], [4]

$$
\hat{\psi} \triangleq \hat{W}^{T} \sigma\left(\hat{V}^{T} \bar{x}_{d}\right)
$$

where $\hat{V}(t) \in \mathbb{R}^{\left(N_{1}+1\right) \times N_{2}}$ and $\hat{W}(t) \in \mathbb{R}^{\left(N_{2}+1\right) \times n}$ are subsequently designed estimates of the ideal weight matrices. The estimate mismatch for the ideal weight matrices are defined as $\tilde{V} \triangleq V-\hat{V}$ and $\tilde{W} \triangleq W-$ $\hat{W}$. The mismatch for the hidden-layer output error $\tilde{\sigma}\left(\bar{x}_{d}\right) \in \mathbb{R}^{N_{2}+1}$ is defined as $\tilde{\sigma} \triangleq \sigma-\hat{\sigma}=\sigma\left(V^{T} \bar{x}_{d}\right)-\sigma\left(\hat{V}^{T} \bar{x}_{d}\right)$. A. 4: the ideal weights are assumed to exist and be bounded by known positive values [3], [4], [32].

Based on the open-loop error system in (5), the control input is composed of a NN estimate term plus the RISE feedback term as [28]

$$
u \triangleq \hat{\psi}+\mu_{1}
$$

where $\hat{\psi}(t) \in \mathbb{R}^{n}$ denotes a subsequently designed, prediction-error based NN feedfoward term. In (10), $\mu_{1}(t) \in \mathbb{R}^{n}$ denotes the RISE feedback term defined generated as [26]-[28]

$$
\dot{\mu}_{1}=\left(k_{1}+1\right) r+\beta_{1} \operatorname{sgn}\left(e_{2}\right), \quad \mu_{1}(0)=-\left(k_{1}+1\right) e_{2}(0)
$$

where $k_{1}, \beta_{1} \in \mathbb{R}$ are positive constant control gains, $\alpha_{2} \in \mathbb{R}$ was introduced in (4). The closed-loop tracking error system can be developed by substituting (10) into (5) as

$$
G^{-1} r=\psi-\hat{\psi}+S_{1}-\mu_{1} .
$$

To facilitate the subsequent composite adaptive control development and stability analysis, (8) and (9) are used to obtain the time derivative of (12), and adding and subtracting the terms $W^{T} \hat{\sigma}^{\prime} \hat{V}^{T} \dot{\bar{x}}_{d}+\hat{W}^{T} \hat{\sigma}^{\prime} \tilde{V}^{T} \dot{\bar{x}}_{d}$ to the resulting expression yields

$$
\begin{aligned}
G^{-1} \dot{r}= & -\dot{G}^{-1} r+\hat{W}^{T} \hat{\sigma}^{\prime} \tilde{V}^{T} \dot{\bar{x}}_{d}+\tilde{W}^{T} \hat{\sigma}^{\prime} \hat{V}^{T} \dot{\bar{x}}_{d} \\
& +W^{T} \sigma^{\prime} V^{T} v_{d}-W^{T} \hat{\sigma}^{\prime} \hat{V}^{T} \dot{\bar{x}}_{d}-\hat{W}^{T} \hat{\sigma}^{\prime} \tilde{V}^{T} \dot{\bar{x}}_{d} \\
& +\dot{S}_{1}-\dot{W}^{T} \hat{\sigma}-\hat{W}^{T} \hat{\sigma}^{\prime} \dot{V}^{T} \bar{x}_{d}+\dot{\varepsilon}-\dot{\mu}_{1} .
\end{aligned}
$$

\section{A. Swapping}

In this section, the swapping procedure is used to generate a measurable form of a prediction error that relates to the function mismatch error (i.e., $\psi(t)-\hat{\psi}(t))$. A measurable form of the prediction error $\eta(t) \in \mathbb{R}^{n}$ is defined as the difference between a filtered control input $u_{f}(t) \in \mathbb{R}^{n}$ and an estimated filtered control input $\hat{u}_{f}(t) \in \mathbb{R}^{n} q$ as

$$
\eta \triangleq u_{f}-\hat{u}_{f}
$$

where the filtered control input is generated from the stable first order differential equation

$$
\dot{u}_{f}+\omega u_{f}=\omega u, \quad u_{f}(0)=0 \quad \text { or } \quad u_{f}=v * u
$$

where $\omega \in \mathbb{R}$ is a known positive constant, " $*$ " is used to denote the standard convolution operation, and the scalar function $v(t) \in \mathbb{R}$ is 
defined as $v \triangleq \omega \exp (-\omega t)$. Using (1), the expression in (15) can be rewritten as

$$
u_{f}=v *\left(G^{-1} \ddot{x}-G^{-1} f\right) .
$$

In (16), the system dynamics in (1) are used to substitute for the control input instead of the its design in (10) in order to force the mismatch $(\psi(t)-\hat{\psi}(t))$ to appear in the prediction error definition (14). The construction of a NN-based controller to approximate the unknown system dynamics in (16) will inherently result in a residual function reconstruction error $\varepsilon\left(\bar{x}_{d}\right)$. To compensate for the effects of the reconstruction error, the typical prediction error formulation is modified to include a RISE-like structure in the design of the estimated filtered control input. Adding and subtracting the term $v *\left(G_{d}^{-1} \ddot{x}_{d}+G_{d}^{-1} f_{d}\right)$ to the expression in (16), and using (6) yields

$$
u_{f}=v *\left(\psi+S-S_{d}\right)
$$

where $S(x, \dot{x}, \ddot{x}), S_{d}\left(x_{d}, \dot{x}_{d}, \ddot{x}_{d}\right) \in \mathbb{R}^{n}$ are defined as

$$
S \triangleq G^{-1} \ddot{x}-G^{-1} f, \quad S_{d} \triangleq G_{d}^{-1} \ddot{x}_{d}-G_{d}^{-1} f_{d} .
$$

The expression in (17) is further simplified as

$$
u_{f}=v * \psi+v * S-v * S_{d} .
$$

The term $v * S(x, \dot{x}, \ddot{x}) \in \mathbb{R}^{n}$ in (19) depends on $\ddot{x}(t)$. Using the following property of convolution [32]:

$$
g_{1} * \dot{g}_{2}=\dot{g}_{1} * g_{2}+g_{1}(0) g_{2}-g_{1} g_{2}(0)
$$

an expression independent of $\ddot{x}(t)$ can be obtained as

$$
v * S=S_{f}+D
$$

where the state-dependent terms are included in the auxiliary function $S_{f}(x, \dot{x}) \in \mathbb{R}^{n}$, defined as

$$
S_{f} \triangleq \dot{v} *\left(G^{-1} \dot{x}\right)+v(0) G^{-1} \dot{x}-v * \dot{G}^{-1} \dot{x}-v * G^{-1} f
$$

and the terms that depend on the initial states are included in $D(t) \in$ $\mathbb{R}^{n}$, defined as

$$
D \triangleq-v G^{-1}(x(0)) \dot{x}(0) \text {. }
$$

Similarly, the expression $v * S_{d}\left(x_{d}, \dot{x}_{d}, \ddot{x}_{d}\right)$ in (19) is evaluated as

$$
v * S_{d}=S_{d f}+D_{d}
$$

where $S_{d f}\left(x_{d}, \dot{x}_{d}\right) \in \mathbb{R}^{n}$ is defined as

$$
S_{d f} \triangleq \dot{v} *\left(G_{d}^{-1} \dot{x}_{d}\right)+v(0) G_{d}^{-1} \dot{x}_{d}-v * \dot{G}_{d}^{-1} \dot{x}_{d}-v * G_{d}^{-1} f_{d}
$$

and $D_{d}(t) \in \mathbb{R}^{n}$ is defined as

$$
D_{d} \triangleq-v G_{d}^{-1}\left(x_{d}(0)\right) \dot{x}_{d}(0) .
$$

Substituting (21)-(26) into (19), and then substituting the resulting expression into (14) yields

$$
\eta=v * \psi+S_{f}-S_{d f}+D-D_{d}-\hat{u}_{f} .
$$

Based on (27) and the subsequent analysis, the filtered control input estimate is designed as

$$
\hat{u}_{f}=\hat{\psi}_{f}+\mu_{2}
$$

where the filtered NN estimate $\hat{\psi}_{f}(t) \in \mathbb{R}^{n}$ is generated from the stable first order differential equation

$$
\dot{\psi}_{f}+\omega \hat{\psi}_{f}=\omega \hat{\psi}, \quad \hat{\psi}_{f}(0)=0
$$

which can be expressed as a convolution as

$$
\hat{\psi}_{f}=v * \hat{\psi} .
$$

In (28), $\mu_{2}(t) \in \mathbb{R}^{n}$ is a RISE-like term generated as

$$
\dot{\mu}_{2} \triangleq k_{2} \eta+\beta_{2} \operatorname{sgn}(\eta), \quad \mu_{2}(0)=0
$$

where $k_{2}, \beta_{2} \in \mathbb{R}$ denote constant positive control gains. In a typical prediction error formulation, the estimated filtered control input is designed to include just the first term $\hat{\psi}_{f}(t)$ in (28). But as discussed earlier, due to the presence of the NN reconstruction error, the unmeasurable form of the prediction error in (27) also includes the filtered reconstruction error. Hence, the estimated filtered control input is augmented with an additional RISE-like term $\mu_{2}(t)$ to cancel the effects of reconstruction error in the prediction error measurement as illustrated in the subsequent design and stability analysis. Substituting (28) into (27) yields the following closed-loop prediction error system:

$$
\eta=v *(\psi-\hat{\psi})+S_{f}-S_{d f}+D-D_{d}-\mu_{2} .
$$

To facilitate the subsequent composite adaptive control development and stability analysis, the time derivative of (31) is expressed as

$$
\dot{\eta}=\dot{v} *(\psi-\hat{\psi})+\omega(\psi-\hat{\psi})+\dot{S}_{f}-\dot{S}_{d f}+\dot{D}-\dot{D}_{d}-\dot{\mu}_{2}
$$

where the property $d / d t(f * g)=(\dot{f} * g)(t)+f(0) g(t)$ was used. Substituting (8) and (9) into (32), subtracting $\dot{v}(t) *\left(\hat{W}^{T} \sigma+\tilde{W}^{T} \hat{\sigma}\right)+$ $\omega\left(\hat{W}^{T} \sigma+\tilde{W}^{T} \hat{\sigma}\right)$ to the resulting expression, and using the Taylor series expansion as in [3] and [4] yields

$$
\begin{aligned}
\dot{\eta}=\tilde{W}^{T}(\hat{\sigma} * \dot{v}+\omega \hat{\sigma})+\hat{W}^{T} \hat{\sigma}^{\prime} \tilde{V}^{T}\left(\bar{x}_{d} * \dot{v}+\omega \bar{x}_{d}\right) \\
+\tilde{N}_{2}+N_{2 B}-k_{2} \eta-\beta_{2} \operatorname{sgn}(\eta)
\end{aligned}
$$

where (30) was utilized. In (33), the unmeasurable/unknown auxiliary term $\tilde{N}_{2}\left(e_{1}, e_{2}, r, t\right) \in \mathbb{R}^{n}$ is defined as

$$
\tilde{N}_{2} \triangleq \dot{S}_{f}-\dot{S}_{d f}
$$

and the term $N_{2 B}(t) \in \mathbb{R}^{n}$ is defined as

$$
\begin{aligned}
N_{2 B} \triangleq \dot{D}-\dot{D}_{d}+\dot{v} * & \left(\hat{W}^{T} O\left(\tilde{V}^{T} \bar{x}_{d}\right)^{2}+\tilde{W}^{T} \tilde{\sigma}+\varepsilon\right) \\
& +\omega\left(\hat{W}^{T} O\left(\tilde{V}^{T} \bar{x}_{d}\right)^{2}+\tilde{W}^{T} \tilde{\sigma}+\varepsilon\right) .
\end{aligned}
$$

In a similar manner as in [27], the Mean Value Theorem can be used to develop the following upper bound for the expression in (34):

$$
\left\|\tilde{N}_{2}(t)\right\| \leq \rho_{2}(\|z\|)\|z\|, \quad z(t) \triangleq\left[\begin{array}{lll}
e_{1}^{T} & e_{2}^{T} & r^{T}
\end{array}\right]^{T}
$$

where the bounding function $\rho_{2}(\cdot) \in \mathbb{R}$ is a positive, globally invertible, nondecreasing function. Using A. 3, and the fact that $v(t)$ is a linear, strictly proper, exponentially stable transfer function, the following inequality can be developed based on the expression in (35) with a similar approach as in Lemma 2 of [15]:

$$
\left\|N_{2 B}(t)\right\| \leq \xi
$$

where $\xi \in \mathbb{R}$ is a known positive constant. 


\section{B. Composite Adaptation}

The composite adaptation for the NN weight estimates is given by

$$
\begin{aligned}
& \dot{\hat{W} \triangleq} \Gamma_{1} \operatorname{proj}\left(\alpha_{2} \hat{\sigma}^{\prime} \hat{V}^{T} \dot{\bar{x}}_{d} e_{2}^{T}+\dot{\hat{\sigma}}_{f} \eta^{T}\right) \\
& \dot{\hat{V}} \triangleq \Gamma_{2} \operatorname{proj}\left(\alpha_{2} \dot{\bar{x}}_{d} e_{2}^{T} \hat{W}^{T} \hat{\sigma}^{\prime}+\dot{\bar{x}}_{d f} \eta^{T} \hat{W}^{T} \hat{\sigma}^{\prime}\right)
\end{aligned}
$$

where $\Gamma_{1} \in \mathbb{R}^{\left(N_{2}+1\right) \times\left(N_{2}+1\right)}, \Gamma_{2} \in \mathbb{R}^{\left(N_{1}+1\right) \times\left(N_{1}+1\right)}$ are constant, positive definite, symmetric control gain matrices, $\operatorname{proj}(\cdot)$ denotes a smooth projection operator (see [20] and [33]) that is used to ensure that $\hat{W}(t)$ and $\hat{V}(t)$ remain inside the bounded convex region. The filtered activation function $\hat{\sigma}_{f}(t) \in \mathbb{R}^{N_{2}+1}$ and the filtered NN input vector $\bar{x}_{d f}(t) \in \mathbb{R}^{3 n+1}$ are given by $\hat{\sigma}_{f}=v * \hat{\sigma}$ and $\bar{x}_{d f}=v * \bar{x}_{d}$, respectively. The projection used in the $\mathrm{NN}$ weight adaptation laws in (38) and (39) can be decomposed into two terms as

$$
\hat{W}=\chi_{\eta}^{W}+\chi_{e_{2}}^{W}, \quad \dot{V}=\chi_{\eta}^{V}+\chi_{e_{2}}^{V}
$$

such that the auxiliary functions $\chi_{\eta}^{W}\left(\hat{\sigma}_{f}, \eta\right), \chi_{e_{2}}^{W}\left(\hat{V}, \bar{x}_{d}, \dot{\bar{x}}_{d}, e_{2}\right) \in$ $\mathbb{R}^{\left(N_{2}+1\right) \times n} \quad$ and $\quad \chi_{\eta}^{V}\left(\bar{x}_{d f}, \hat{W}, \hat{V}, \eta\right), \quad \chi_{e_{2}}^{V}\left(\hat{W}, \hat{V}, \bar{x}_{d}, \dot{\bar{x}}_{d}, e_{2}\right) \quad \in$ $\mathbb{R}^{\left(N_{1}+1\right) \times N_{2}}$ satisfy the following bounds:

$$
\begin{aligned}
\left\|\chi_{\eta}^{W}\right\| & \leq b_{1}\|\eta\|, \quad\left\|\chi_{e_{2}}^{W}\right\| \leq b_{2}\left\|e_{2}\right\| \\
\left\|\chi_{\eta}^{V}\right\| \leq b_{1}^{\prime}\|\eta\|, & \left\|\chi_{e_{2}}^{V}\right\| \leq b_{2}^{\prime}\left\|e_{2}\right\|
\end{aligned}
$$

where $b_{1}, b_{2}, b_{1}^{\prime}$, and $b_{2}^{\prime} \in \mathbb{R}$ are known positive constants. To facilitate the subsequent stability analysis, the following inequality is developed based on (41) and the fact that the $\mathrm{NN}$ weight estimates are bounded by the smooth projection algorithm

$$
\left\|\chi_{\eta}^{W} \hat{\sigma}+\hat{W}^{T} \hat{\sigma}^{\prime} \chi_{\eta}^{V} \bar{x}_{d}\right\| \leq c_{1}\|\eta\|
$$

where $c_{1} \in \mathbb{R}$ is a positive constant.

\section{Closed-Loop Error System}

Substituting for $\hat{W}(t)$ and $\hat{V}(t)$ from (40), the expression in (13) can be rewritten as

$$
\begin{aligned}
G^{-1} \dot{r}=-\frac{1}{2} \dot{G}^{-1} r-\chi_{\eta}^{W} \hat{\sigma}- & \hat{W}^{T} \hat{\sigma}^{\prime} \chi_{\eta}^{V} \bar{x}_{d}+\tilde{N}_{1}+N_{1} \\
& -\left(k_{1}+1\right) r-\beta_{1} \operatorname{sgn}\left(e_{2}\right)-e_{2} .
\end{aligned}
$$

In (43), the unmeasurable/unknown auxiliary terms $\tilde{N}_{1}\left(e_{1}, e_{2}, r, t\right)$ and $N_{1}(t) \in \mathbb{R}^{n}$ are defined as

$$
\begin{aligned}
& \tilde{N}_{1} \triangleq-\frac{1}{2} \dot{G}^{-1} r+\dot{S}_{1}+e_{2}-\chi_{e_{2}}^{W} \hat{\sigma}-\hat{W}^{T} \hat{\sigma}^{\prime} \chi_{e_{2}}^{V} \bar{x}_{d} \\
& N_{1} \triangleq N_{d}+N_{1 B} .
\end{aligned}
$$

In (45) $N_{d}\left(\bar{x}_{d}, \dot{\bar{x}}_{d}, t\right)$ and $N_{1 B}\left(\hat{W}, \hat{V}, \bar{x}_{d}, \dot{\bar{x}}_{d}, t\right) \in \mathbb{R}^{n}$ are defined as

$$
N_{d} \triangleq W^{T} \sigma^{\prime} V^{T} \dot{\bar{x}}_{d}+\dot{\varepsilon}, \quad N_{1 B} \triangleq N_{1 B_{a}}+N_{1 B_{b}}
$$

where $N_{1 B_{a}}\left(\hat{W}, \hat{V}, \bar{x}_{d}, \dot{\bar{x}}_{d}, t\right) \in \mathbb{R}^{n}$ and $N_{1 B_{b}}\left(\hat{W}, \hat{V}, \bar{x}_{d}, \dot{\bar{x}}_{d}, t\right) \in$ $\mathbb{R}^{n}$ are defined as

$$
\begin{aligned}
& N_{1 B_{a}} \triangleq-W^{T} \hat{\sigma}^{\prime} \hat{V}^{T} \dot{\bar{x}}_{d}-\hat{W}^{T} \hat{\sigma}^{\prime} \tilde{V}^{T} \dot{\bar{x}}_{d} \\
& N_{1 B_{b}} \triangleq \hat{W}^{T} \hat{\sigma}^{\prime} \tilde{V}^{T} \dot{\bar{x}}_{d}+\tilde{W}^{T} \hat{\sigma}^{\prime} \hat{V}^{T} \dot{\bar{x}}_{d} .
\end{aligned}
$$

Motivation for segregating the terms in (45) is derived from the fact that the different components in (45) have different bounds. Segregating the terms as in (45)-(47) facilitates the development of the NN weight update laws and the subsequent stability analysis. For example, the terms in $N_{d}(t)$ are grouped together because the terms and their time derivatives can be upper bounded by a constant and rejected by the RISE feedback, whereas the terms grouped in $N_{1 B}(t)$ can be upper bounded by a constant but their derivatives are state dependent. The state dependency of the term $\dot{N}_{1 B}(t)$ violates the assumptions given in previous RISE-based controllers (e.g., [25], [27], [29]), and requires additional consideration in the adaptation law design and stability analysis. The terms in $N_{1 B}(t)$ are further segregated because $N_{1 B_{a}}\left(\hat{W}, \hat{V}, \bar{x}_{d}, \dot{\bar{x}}_{d}\right)$ will be rejected by the RISE feedback, whereas $N_{1 B_{b}}\left(\hat{W}, \hat{V}, \bar{x}_{d}, \dot{\bar{x}}_{d}\right)$ will be partially rejected by the RISE feedback and partially canceled by the adaptive update law for the NN weight estimates. In a similar manner as in (36), the following upper bound is developed for the expression in (44):

$$
\left\|\tilde{N}_{1}(t)\right\| \leq \rho_{1}(\|z\|)\|z\|
$$

where the bounding function $\rho_{1}(\cdot) \in \mathbb{R}$ is a positive, globally invertible, nondecreasing function. The following inequalities can be developed based on A. 3, A. 4, (46), and (47):

$$
\left\|N_{d}\right\| \leq \zeta_{1}, \quad\left\|N_{1 B_{a}}\right\| \leq \zeta_{2}, \quad\left\|N_{1 B_{b}}\right\| \leq \zeta_{3}, \quad\left\|\dot{N}_{d}\right\| \leq \zeta_{4} .
$$

From (45), (46), and (49), the following bound can be developed

$$
\left\|N_{1}\right\| \leq\left\|N_{d}\right\|+\left\|N_{1 B}\right\| \leq \zeta_{1}+\zeta_{2}+\zeta_{3} .
$$

By using (38) and (39), the time derivative of $N_{1 B}\left(\hat{W}, \hat{V}, x_{d}\right)$ can be bounded as

$$
\left\|\dot{N}_{1 B}\right\| \leq \zeta_{5}+\zeta_{6}\left\|e_{2}\right\|+\zeta_{7}\|\eta\|
$$

In (49) and (51), $\zeta_{i} \in \mathbb{R},(i=1,2, \ldots, 7)$ are known positive constants.

Remark 1: If the bounds in (49) and (51) are unknown, then a method similar to [31] to can be used to generate an estimate for these bounds by designing an update law. These estimates can be used in the gain conditions for the control development and stability analysis. With additional terms in the Lyapunov function containing the estimates, stability similar to the current result can be achieved.

\section{STABILITY ANALYSIS}

Consider the composite vector $y(t) \in \mathbb{R}^{4 n+3}$ defined as

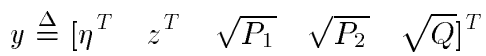

where $\eta(t)$ and $z(t)$ are defined in (14) and (36), respectively. In (52), the auxiliary function $P_{1}(t)$ is defined as

$$
P_{1}(t) \triangleq \beta_{1} \sum_{i=1}^{n}\left|e_{2 i}(0)\right|-e_{2}(0)^{T} N_{1}(0)-\int_{0}^{t} L_{1}(\tau) d \tau
$$

where $e_{2 i}(0) \in \mathbb{R}$ denotes the $i$ th element of the vector $e_{2}(0)$, and the auxiliary function $L_{1}(t) \in \mathbb{R}$ is defined as

$$
L_{1} \triangleq r^{T}\left(N_{1 B_{a}}+N_{d}-\beta_{1} \operatorname{sgn}\left(e_{2}\right)\right)+\dot{e}_{2}^{T} N_{1 B_{b}}-\beta_{3}\left\|e_{2}\right\|^{2}-\beta_{4}\|\eta\|^{2}
$$

where $\beta_{1}, \beta_{3}$, and $\beta_{4} \in \mathbb{R}$ are positive constants chosen according to the sufficient conditions

$$
\begin{aligned}
& \beta_{1}>\max \left\{\zeta_{1}+\zeta_{2}+\zeta_{3}, \zeta_{1}+\zeta_{2}+\frac{\zeta_{4}}{\alpha_{2}}+\frac{\zeta_{5}}{\alpha_{2}}\right\} \\
& \beta_{3}>\zeta_{6} \frac{\zeta_{7}}{2}, \quad \beta_{4}>\frac{\zeta_{7}}{2}
\end{aligned}
$$


where $\zeta_{1}, \zeta_{2}, \ldots, \zeta_{7}$ were introduced in (49)-(51). If the sufficient conditions introduced in (55) are satisfied, the following inequality is obtained [27], [34]:

$$
\int_{0}^{t} L_{1}(\tau) d \tau \leq \beta_{1} \sum_{i=1}^{n}\left|e_{2 i}(0)\right|-e_{2}(0)^{T} N_{1}(0)
$$

Hence, (56) can be used to conclude that $P_{1}(t) \geq 0$. Also in (52), the auxiliary function $P_{2}(t)$ is defined as

$$
P_{2}(t) \triangleq-\int_{0}^{t} L_{2}(\tau) d \tau, \quad L_{2} \triangleq \eta^{T}\left(N_{2 B}-\beta_{2} \operatorname{sgn}(\eta)\right)
$$

where $\beta_{2} \in \mathbb{R}$ is a positive constant chosen according to the sufficient condition

$$
\beta_{2}>\xi
$$

where $\xi$ was introduced in (37). Provided the sufficient condition introduced in (58) is satisfied, then $P_{2}(t) \geq 0$. The auxiliary function $Q(t)$ in (52) is defined as

$$
Q(t) \triangleq \frac{1}{2} \operatorname{tr}\left(\tilde{W}^{T} \Gamma_{1}^{-1} \tilde{W}\right)+\frac{1}{2} \operatorname{tr}\left(\tilde{V}^{T} \Gamma_{2}^{-1} \tilde{V}\right), \quad Q(t) \geq 0 .
$$

Remark 2: From (4), (33), (43), (53), (54), and (57), some of the differential equations describing the closed-loop system have discontinuous right-hand sides. The existence and uniqueness of the solutions to the discontinuous differential equations is understood in the Filippov sense [35], [36].

To facilitate the subsequent stability analysis, let $\mathcal{D} \subset \mathbb{R}^{4 n+3}$ be a domain containing $y(t)=0$ defined as

$$
\mathcal{D} \triangleq\left\{y(t) \in \mathbb{R}^{4 n+3} \mid\|y\| \leq \rho^{-1}\left(2 \sqrt{\lambda_{3} k}\right)\right\} .
$$

In (60), $\lambda_{3} \in \mathbb{R}$ denotes a positive constant defined as

$$
\lambda_{3} \triangleq \min \left\{\alpha_{1}-\frac{1}{2}, \alpha_{2}-\frac{1}{2}-\beta_{3}, 1\right\} \text { s.t. } \alpha_{1}>\frac{1}{2}, \alpha_{2}>\beta_{3}+\frac{1}{2} \text {. }
$$

Theorem: The controller given in (9)-(11) in conjunction with the composite NN adaptation laws in (38) and (39), where the prediction error is generated from (14), (15), (28)-(30), ensures that all system signals are bounded under closed-loop operation and that the position tracking error and the prediction error are regulated, provided the sufficient conditions in (55), (58) and (61) are satisfied, in the sense that

$$
\left\|e_{1}(t)\right\| \rightarrow 0 \text { and } \quad\|\eta(t)\| \rightarrow 0 \quad \text { as } \quad t \rightarrow \infty
$$

for all $y(0)$ in a bounded compact set $\mathcal{S} \subset \mathcal{D}$. The set $\mathcal{S}$ can be made arbitrarily large by selecting the control gains $k_{1}$ and $k_{2}$ introduced in (11) and (30) based on the initial conditions of the system (i.e., a semi-global result).

Proof: Let $V_{L}(y, t): \mathcal{D} \times[0, \infty) \rightarrow \mathbb{R}$ be a continuously differentiable, positive definite function defined as

$$
V_{L} \triangleq \frac{1}{2} e_{1}^{T} e_{1}+\frac{1}{2} e_{2}^{T} e_{2}+\frac{1}{2} r^{T} G^{-1} r+\frac{1}{2} \eta^{T} \eta+P_{1}+P_{2}+Q
$$

which satisfies the inequalities

$$
U_{1}(y) \leq V_{L}(y, t) \leq U_{2}(y)
$$

provided the sufficient conditions introduced in (55) and (58) are satisfied. In (63), the continuous positive definite functions $U_{1}(y), U_{2}(y) \in$ $\mathbb{R}$ are defined as $U_{1}(y) \triangleq \lambda_{1}\|y\|^{2}$ and $U_{2}(y) \triangleq \lambda_{2}(x)\|y\|^{2}$, where $\lambda_{1}, \lambda_{2}(x) \in \mathbb{R}$ are defined as $\lambda_{1} \triangleq(1 / 2) \min \{1, g\}$ and $\lambda_{2} \triangleq$ $\max \{(1 / 2) \bar{g}(x), 1\}$, respectively, where $g, \bar{g}(x)$ are introduced in (2). Using (4), (33), (43), (45), (46), (53), (54), and (57), the time derivative of (62) can be expressed as

$$
\begin{aligned}
\dot{V}_{L}= & e_{1}^{T} e_{2}-\alpha_{1} e_{1}^{T} e_{1}-\alpha_{2} e_{2}^{T} e_{2}-k_{2} \eta^{T} \eta-r^{T} \chi_{\eta}^{W} \hat{\sigma} \\
& -r^{T} \hat{W}^{T} \hat{\sigma}^{\prime} \chi_{\eta}^{V} \bar{x}_{d}+r^{T} \tilde{N}_{1}-\left(k_{1}+1\right) r^{T} r+\beta_{4}\|\eta\|^{2} \\
& +\eta^{T} \tilde{N}_{2}+\operatorname{tr}\left(\alpha_{2} \tilde{W}^{T} \hat{\sigma}^{\prime} \hat{V}^{T} \dot{\bar{x}}_{d} e_{2}^{T}\right)-\operatorname{tr}\left(\tilde{V}^{T} \Gamma_{2}^{-1} \dot{\hat{V}}\right) \\
& +\operatorname{tr}\left(\tilde{W}^{T} \dot{\hat{\sigma}}_{f} \eta^{T}\right)+\operatorname{tr}\left(\alpha_{2} \tilde{V}^{T} \dot{\bar{x}}_{d}\left(\hat{\sigma}^{\prime T} \hat{W}_{e_{2}}\right)^{T}\right) \\
& +\operatorname{tr}\left(\tilde{V}^{T} \dot{\bar{x}}_{d f}\left(\hat{\sigma}^{\prime} T \hat{W} \eta\right)^{T}\right)-\operatorname{tr}\left(\tilde{W}^{T} \Gamma_{1}^{-1} \dot{\hat{W}}\right) \\
& +\beta_{3}\left\|e_{2}\right\|^{2} .
\end{aligned}
$$

Substituting the update laws from (38) and (39) in (64), canceling the similar terms, and using the fact that $e_{1}^{T} e_{2} \leq(1 / 2)\left(\left\|e_{1}\right\|^{2}+\left\|e_{2}\right\|^{2}\right)$, the expression in (64) is upper bounded as

$$
\begin{aligned}
\dot{V}_{L} \leq-\lambda_{3}\|z\|^{2}-k_{1}\|r\|^{2} & +\|r\|\left\|\tilde{N}_{1}\right\| \\
& +c_{1}\|\eta\|\|z\|+\|\eta\|\left\|\tilde{N}_{2}\right\|-k_{2}\|\eta\|^{2} .
\end{aligned}
$$

Letting $k_{2}=k_{2 a}+k_{2 b}$, where $k_{2 a}, k_{2 b} \in \mathbb{R}$ are positive constants, and using the inequalities in (36) and (48), the expression in (65) is upper bounded as

$$
\begin{aligned}
\dot{V}_{L} \leq-\left(k_{2 b}-\beta_{4}\right)\|\eta\|^{2}-\left[k_{1}\|r\|^{2}-\rho_{1}(\|z\|)\|r\|\|z\|\right] \\
-\lambda_{3}\|z\|^{2}-\left[k_{2 a}\|\eta\|^{2}-\left(\rho_{2}(\|z\|)+c_{1}\right)\|\eta\|\|z\|\right] .
\end{aligned}
$$

Completing the squares for the terms inside the brackets in (66) yields

$$
\begin{aligned}
\dot{V}_{L} & \leq-\lambda_{3}\|z\|^{2}+\frac{\rho^{2}(\|z\|)\|z\|^{2}}{4 k}-\left(k_{2 b}-\beta_{4}\right)\|\eta\|^{2} \\
& \leq-U(y)
\end{aligned}
$$

where $k \triangleq \min \left\{k_{1}, k_{2 a}\right\}$ and $\rho(\cdot) \in \mathbb{R}$ is a positive, globally invertible, nondecreasing function defined as

$$
\rho^{2}(\|z\|) \triangleq \rho_{1}^{2}(\|z\|)+\left(\rho_{2}(\|z\|)+c_{1}\right)^{2} .
$$

In (67), $U(y)=c\left\|\left[z^{T} \eta^{T}\right]^{T}\right\|^{2}$, for some positive constant $c$, is a continuous, positive semi-definite function that is defined on the domain $\mathcal{D}$.

The inequalities in (63) and (67) can be used to show that $V_{L}(y, t) \in$ $\mathcal{L}_{\infty}$ in $\mathcal{D}$; hence, $e_{1}(t), e_{2}(t), r(t)$, and $\eta(t) \in \mathcal{L}_{\infty}$ in $\mathcal{D}$ and $\dot{e}_{1}(t)$, and $\dot{e}_{2}(t) \in \mathcal{L}_{\infty}$ in $\mathcal{D}$ from (4). Therefore, A. 3 can be used along with (3), (4) to conclude that $x^{(i)}(t) \in \mathcal{L}_{\infty}$, in $\mathcal{D}$. Since $x^{(i)}(t) \in \mathcal{L}_{\infty}$, in $\mathcal{D}$, A. 2 can be used to conclude that $G^{-1}(\cdot)$ and $f(\cdot) \in \mathcal{L}_{\infty}$ in $\mathcal{D}$. Thus, from (1) we can show that $u(t) \in \mathcal{L}_{\infty}$ in $\mathcal{D}$. Therefore, $u_{f}(t) \in \mathcal{L}_{\infty}$ in $\mathcal{D}$, and hence, from (14), $\hat{u}_{f}(t) \in \mathcal{L}_{\infty}$ in $\mathcal{D}$. Given that $r(t) \in \mathcal{L}_{\infty}$ in $\mathcal{D},(11)$ can be used to show that $\dot{\mu}_{1}(t) \in \mathcal{L}_{\infty}$ in $\mathcal{D}$, and since $\dot{G}^{-1}(\cdot)$ and $\dot{f}(\cdot) \in \mathcal{L}_{\infty}$ in $\mathcal{D},(43)$ can be used to show that $\dot{r}(t) \in \mathcal{L}_{\infty}$ in $\mathcal{D}$, and (33) can be used to show that $\dot{\eta}(t) \in \mathcal{L}_{\infty}$ in $\mathcal{D}$. Since $\dot{e}_{1}(t), \dot{e}_{2}(t)$, $\dot{r}(t)$, and $\dot{\eta}(t) \in \mathcal{L}_{\infty}$ in $\mathcal{D}$, the definitions for $U(y)$ and $z(t)$ can be used to prove that $U(y)$ is uniformly continuous in $\mathcal{D}$.

Let $\mathcal{S} \subset \mathcal{D}$ denote a set defined as

$$
\mathcal{S} \triangleq\left\{y(t) \in \mathcal{D} \mid U_{2}(y(t))<_{1}\left(\rho^{-1}\left(2 \sqrt{\lambda_{3} k}\right)\right)^{2}\right\}
$$

which can be made arbitrarily large to include any initial conditions by increasing the control gain $k$ (i.e., a semi-global stability result). Theorem 8.4 of [37] can now be invoked to state that

$$
c\left\|\left[\begin{array}{ll}
z^{T} & \eta^{T}
\end{array}\right]^{T}\right\|^{2} \rightarrow 0 \quad \text { as } \quad t \rightarrow \infty \quad \forall y(0) \in \mathcal{S} .
$$



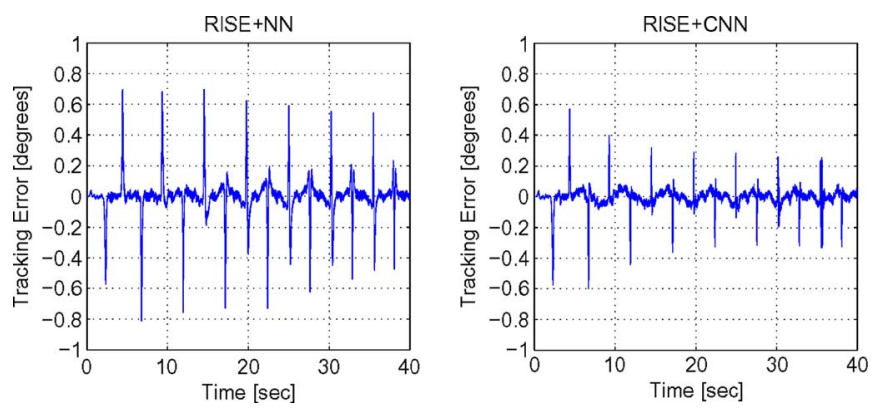

Fig. 1. Tracking errors for the RISE+NN and the RISE+CNN controllers.
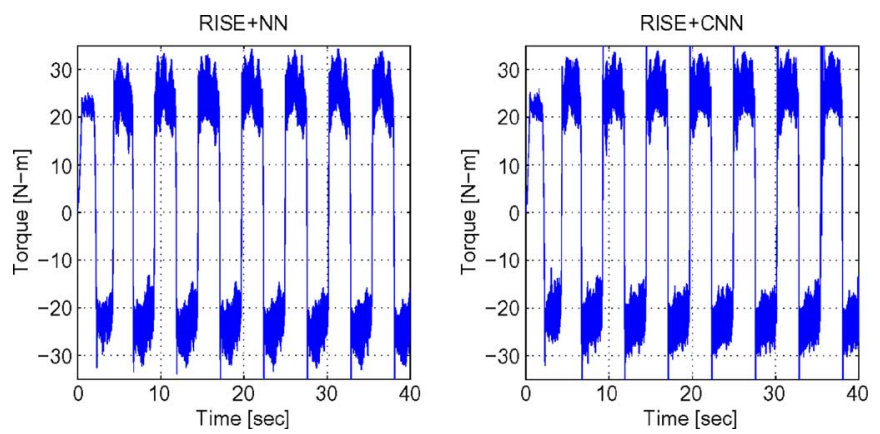

Fig. 2. Torques for the RISE+NN and the RISE $+\mathrm{CNN}$ controllers.

Based on the definition of $z(t),(69)$ can be used to show that

$$
\left\|e_{1}(t)\right\|,\|\eta(t)\| \rightarrow 0 \quad \text { as } \quad t \rightarrow \infty \quad \forall y(0) \in \mathcal{S} .
$$

\section{EXPERIMENTAL RESULTS}

A testbed was used to implement the developed controller. The testbed consists of a circular disc of unknown inertia mounted on a direct-drive switched reluctance motor. A rectangular nylon block was mounted on a pneumatic linear thruster to apply an external friction load of 15 psi to the rotating disk. The dynamics for the testbed are given as

$$
J \ddot{q}+f(\dot{q})+\tau_{d}(t)=\tau(t)
$$

where $J \in \mathbb{R}$ denotes the combined inertia of the circular disk and rotor assembly, $f(\dot{q}) \in \mathbb{R}$ denotes the friction torque, $\tau_{d}(t) \in \mathbb{R}$ denotes a general nonlinear disturbance (e.g., unmodeled effects), and $\tau(t) \in \mathbb{R}$ denotes the control torque input. The desired link trajectory was selected as (in degrees) $q_{d}(t)=60.0 \sin (1.2 t)\left(1-\exp \left(-0.01 t^{3}\right)\right)$. The following control gains were used:

$$
\begin{aligned}
& k_{1}=70, \quad \beta_{1}=50, \quad \alpha_{1}=20, \quad \alpha_{2}=10, \quad \Gamma_{1}=20 I_{11} \\
& \Gamma_{2}=I_{4} \quad k_{2}=70, \quad \beta_{2}=50, \quad \omega=8 .
\end{aligned}
$$

Two different experiments were conducted, first without (RISE+NN) and second with (RISE+CNN) the prediction error component of the update laws in (38) and (39). The tracking errors and torques are shown in Fig. 1 and Fig. 2, respectively. Each experiment was performed five times and the average RMS error and torque values were calculated. The average RMS tracking error (in deg) for the RISE+NN controller is 0.135 , compared to 0.071 for the RISE+CNN (proposed) controller. The average RMS torques (in N-m) for the respective controllers is 24.01 and 23.77, which indicate that the proposed RISE+CNN controller yields a lower RMS error with a similar control effort. The experimental results indicates high frequency content in the tracking error and control input, but since the proposed controller implements an integral of the $\operatorname{sgn}(\cdot)$ function, it does not exhibit instantaneous switching like a discontinuous sliding mode controller.

\section{CONCLUSION}

A novel gradient-based composite NN controller is developed for nonlinear uncertain systems, where the $\mathrm{NN}$ weight estimates are generated using a composite update law driven by both the tracking and the prediction error. The construction of a NN-based controller to approximate the unknown system dynamics inherently results in a residual function reconstruction error, which has been the technical obstacle that has prevented the development of composite adaptation laws for NNs. To compensate for the effects of the reconstruction error, a RISE-based swapping procedure is presented.

\section{REFERENCES}

[1] R. Ortega, Passivity-Based Control of Euler-Lagrange Systems: Mechanical, Electrical, and Electromechanical Applications. Berlin/New York: Springer-Verlag, 1998.

[2] K. Hornik, M. Stinchcombe, and H. White, "Multilayer feedforward networks are universal approximators," Neural Netw., vol. 2, pp. 359-366, 1985

[3] F. L. Lewis, "Nonlinear network structures for feedback control," Asian J. Control, vol. 1, no. 4, pp. 205-228, 1999.

[4] F. Lewis, J. Campos, and R. Selmic, Neuro-Fuzzy Control of Industrial Systems With Actuator Nonlinearities. Philadelphia, PA: SIAM, 2002.

[5] R. Sanner and J. Slotine, "Gaussian networks for direct adaptive control," IEEE Trans. Neural Netw., vol. 3, no. 6, pp. 837-863, Nov. 1992.

[6] R. Sanner and J. Slotine, "Stable adaptive control of robot manipulators using neural networks," Neural Comput., vol. 7, no. 4, pp. 753-790, 1995.

[7] E. Tzirkel-Hancock and F. Fallside, Stable Control of Nonlinear Systems Using Neural Networks Dept. Eng., Univ. Cambridge. Cambridge, U.K., 1991.

[8] F. Chen and H. Khalil, "Adaptive control of a class of nonlinear discrete-time systems using neural networks," IEEE Trans. Autom. Control, vol. 40, no. 5, pp. 791-801, May 1995.

[9] S. Jagannathan and F. Lewis, "Multilayer discrete-time neural-net controller with guaranteed performance," IEEE Trans. Neural Netw., vol. 7, no. 1, pp. 107-130, Jan. 1996.

[10] S. Fabri and V. Kadirkamanathan, "Dynamic structure neural networks for stable adaptive control of nonlinear systems," IEEE Trans. Neural Netw., vol. 7, no. 5, pp. 1151-1167, Sep. 1996.

[11] S. Ge, T. Lee, and C. Harris, Adaptive Neural Network Control of Robotic Manipulators. Singapore: World Scientific, 1998.

[12] A. Morse, "Global stability of parameter-adaptive control systems," IEEE Trans. Autom. Control, vol. AC-25, no. 3, pp. 433-439, Jun. 1980.

[13] J.-B. Pomet and L. Praly, "Indirect adaptive nonlinear control," in Proc IEEE Conf. Decision and Control, Dec. 7-9, 1988, pp. 2414-2415.

[14] S. Sastry and A. Isidori, "Adaptive control of linearizable systems," IEEE Trans. Autom. Control, vol. 34, no. 11, pp. 1123-1131, Nov. 1989.

[15] R. H. Middleton and C. G. Goodwin, "Adaptive computed torque control for rigid link manipulators," Syst. Control Lett., vol. 10, pp. 9-16, 1988.

[16] J. J. Slotine and W. Li, "Composite adaptive control of robot manipulators," Automatica, vol. 25, no. 4, pp. 509-519, Jul. 1989.

[17] J. J. Slotine and W. Li, Applied Nonlinear Control. Upper Saddle River, NJ: Prentice-Hall, 1991.

[18] M. S. de Queiroz, D. M. Dawson, and M. Agarwal, "Adaptive control of robot manipulators with controller/update law modularity," $\mathrm{Au}$ tomatica, vol. 35, pp. 1379-1390, 1999. 
[19] E. Zergeroglu, W. Dixon, D. Haste, and D. Dawson, "A composite adaptive output feedback tracking controller for robotic manipulators," in Proc. American Control Conf., 1999, pp. 3013-3017.

[20] M. Krstic, I. Kanellakopoulos, and P. Kokotovic, Nonlinear and Adaptive Control Design. New York: Wiley, 1995.

[21] S. Fabri and V. Kadirkamanathan, "Neural control of nonlinear systems with composite adaptation for improved convergence of Gaussian networks," in Proc. European Control Conf., Brussels, Belgium, 1997.

[22] S. Fabri and V. Kadirkamanathan, Functional Adaptive Control: An Intelligent Systems Approach. London, U.K.: Springer-Verlag, 2001.

[23] B. Peterson and K. Narendra, "Bounded error adaptive control," IEEE Trans. Autom. Control, vol. AC-27, no. 6, pp. 1161-1168, Dec. 1982.

[24] J. Nakanishi, J. Farrell, and S. Schaal, "Composite adaptive control with locally weighted statistical learning," Neural Netw., vol. 18, no. 1, pp. 71-90, 2005.

[25] P. M. Patre, W. MacKunis, C. Makkar, and W. E. Dixon, "Asymptotic tracking for systems with structured and unstructured uncertainties," IEEE Trans. Control Syst. Technol., vol. 16, no. 2, pp. 373-379, Mar. 2008.

[26] Z. Qu and J. Xu, "Model-based learning controls and their comparisons using Lyapunov direct method," Asian J. Control, vol. 4, no. 1, pp. 99-110, Mar. 2002.

[27] B. Xian, D. M. Dawson, M. S. de Queiroz, and J. Chen, "A continuous asymptotic tracking control strategy for uncertain nonlinear systems," IEEE Trans. Autom. Control, vol. 49, no. 7, pp. 1206-1211, Jul. 2004.

[28] P. M. Patre, W. MacKunis, K. Kaiser, and W. E. Dixon, "Asymptotic tracking for uncertain dynamic systems via a multilayer neural network feedforward and RISE feedback control structure," IEEE Trans. Autom. Control, vol. 53, no. 9, pp. 2180-2185, Oct. 2008.

[29] Z. Cai, M. S. de Queiroz, and D. M. Dawson, "Robust adaptive asymptotic tracking of nonlinear systems with additive disturbance," IEEE Trans. Autom. Control, vol. 51, pp. 524-529, Mar. 2006.

[30] B. Xian, M. S. de Queiroz, and D. M. Dawson, A Continuous Control Mechanism for Uncertain Nonlinear Systems in Optimal Control, Stabilization, and Nonsmooth Analysis. Heidelberg, Germany: SpringerVerlag, 2004.

[31] M. Polycarpou, "Stable adaptive neural control scheme for nonlinear systems," IEEE Trans. Autom. Control, vol. 41, no. 3, pp. 447-451, Mar. 1996.

[32] F. L. Lewis, C. Abdallah, and D. Dawson, Control of Robot Manipulators. New York: MacMillan, 1993.

[33] W. E. Dixon, A. Behal, D. M. Dawson, and S. P. Nagarkatti, Nonlinear Control of Engineering Systems: A Lyapunov-Based Approach. Boston, MA: Birkhäuser, 2003.

[34] P. M. Patre, W. MacKunis, C. Makkar, and W. E. Dixon, "Asymptotic tracking for systems with structured and unstructured uncertainties," in Proc. IEEE Conf. Decision and Control, San Diego, CA, 2006, pp. 441-446.

[35] M. M. Polycarpou and P. A. Ioannou, "On the existence and uniqueness of solutions in adaptive control systems," IEEE Trans. Autom. Control, vol. 38, no. 3, pp. 474-479, Mar. 1993.

[36] Z. Qu, Robust Control of Nonlinear Uncertain Systems. New-York: Wiley, 1998.

[37] H. K. Khalil, Nonlinear Systems, 3rd ed. Upper Saddle River, NJ: Prentice-Hall, 2002.

\section{Finite-Time Consensus Problems for Networks of Dynamic Agents}

\author{
Long Wang and Feng Xiao
}

Abstract-In this note, we discuss finite-time state consensus problems for multi-agent systems and present one framework for constructing effective distributed protocols, which are continuous state feedbacks. By employing the theory of finite-time stability, we investigate both the bidirectional interaction case and the unidirectional interaction case, and prove that if the sum of time intervals, in which the interaction topology is connected, is sufficiently large, the proposed protocols will solve the finite-time consensus problems.

Index Terms-Distributed control, finite-time consensus, multi-agent systems, time-varying topologies.

\section{INTRODUCTION}

The consensus theory of multi-agent systems has emerged as a challenging new area of research in recent years [1]. It is a basic and fundamental research topic in decentralized control of networks of dynamic agents and has attracted great attention of researchers. This is partly due to its broad applications in cooperative control of unmanned air vehicles, formation control of mobile robots, control of communication networks, design of sensor networks, flocking of social insects, swarm-based computing, etc.

In the analysis of consensus problems, convergence rate is an important performance indicator for the proposed consensus protocol. It was shown that the second smallest eigenvalue of the interaction graph Laplacian, called algebraic connectivity, quantifies the convergence rate under the typical protocol presented in [2]. To get high convergence rate, several researchers endeavored to find proper interaction graphs with larger algebraic connectivity. In [3], Kim and Mesbahi considered the problem of finding the best vertex positional configuration so that the algebraic connectivity of the associated interaction graph is maximized, where the weight for the edge between any two vertices was assumed to be a function of the distance between the two corresponding agents. In [4], Xiao and Boyd considered and solved the problem of weight design by using semi-definite convex programming, and the convergence rate is also increased. Simulation results showed that the interaction graph with small-world property possesses large algebraic connectivity [5]. However, it can be observed that all those efforts were to choose proper interaction graphs, but not to find available protocols with high performance. On the other hand, although by maximizing the algebraic connectivity of interaction graph, we can increase convergence rate with respect to the linear protocol proposed in [2], the state consensus can never occur in finite time. In practice, it is often required that the consensus be reached in a finite time. And there are a number of situations, in which finite-time convergence is

Manuscript received January 26, 2007; revised March 12, 2009 and August 17, 2009. First published February 02, 2010; current version published April 02, 2010. This work was supported by the National Science Foundation of China (NSFC) under Grants 60904062, 10972002, and 60925011. Recommended by Associate Editor Z. Qu.

L. Wang is with the Intelligent Control Laboratory, Center for Systems and Control, College of Engineering, and Key Laboratory of Machine Perception (Ministry of Education), Peking University, Beijing 100871, China (e-mail: longwang@pku.edu.cn; longwang@mech.pku.edu.cn).

F. Xiao is now with the School of Automation, Beijing Institute of Technology, Beijing 100081, China (e-mail: fengxiao@pku.edu.cn).

Digital Object Identifier 10.1109/TAC.2010.2041610 